\title{
Biopterin derivatives in normal and phenylketonuric patients after oral loads of $\mathrm{L}$-phenylalanine, L-tyrosine, and L-tryptophan
}

\author{
R. J. LEEMING, J. A. BLAIR, ANNE GREEN, and D. N. RAINE \\ From the General Hospital, University of Aston, and the Children's Hospital, Birmingham
}

Leeming, R. J., Blair, J. A., Green, A., and Raine, D. N. (1976). Archives of Disease in Childhood, 51, 771. Biopterin derivatives in normal and phenylketonuric patients after oral loads of L-phenylalanine, L-tyrosine, and L-tryptophan. Plasma biopterin derivatives studied in 10 normal and 21 phenylketonuric children showed a significantly high concentration in the latter group. Biopterin derivatives correlated with plasma phenylalanine concentration, but in normal adults given an oral phenylalanine load the rate of increase with phenylalanine differed from that in phenylketonuric patients.

A patient with hyperphenylalaninaemia, not due to phenylketonuria, had an abnormal biopterin derivatives response to phenylalanine distinct from that of patients with classical phenylketonuria. This may be a useful investigation to differentiate some variants of phenylketonuria.

Patients with hyperphenylalaninaemia do not necessarily have phenylketonuria, due to an almost total deficiency of the hepatic enzyme phenylalanine hydroxylase, and diagnosis is complicated by the several variants or atypical forms of phenylketonuria. These variants are currently identified by criteria including plasma phenylalanine concentrations lower than those found in classical phenylketonuria; an increased tolerance to administered phenylalanine over a few hours; and the ability of a patient to sustain a diet containing about 250$500 \mathrm{mg}$ phenylalanine per day rather than 150$250 \mathrm{mg} /$ day in classical phenylketonuria.

Several cases of a new variant have recently been described (Smith, Clayton, and Wolff, 1975; Bartholomé, 1974; Butler et al., 1975; Leeming, Blair, and Rey, 1976a) in which hyperphenylalaninaemia was associated with a progressive neurological illness unlike that seen in untreated classical phenylketonuria. The patients did not respond clinically to a low phenylalanine diet and phenylalanine hydroxylase activity in the liver was normal. Though the nature of the defect in these variants is unknown, it may be due to a defect in the metabolism of biopterin.

Received 19 January 1976.
Tetrahydrobiopterin functions as cofactor in the metabolism of phenylalanine to tyrosine (Blakley, 1969). In this reaction tetrahydrobiopterin donates hydrogen ions and is converted to quinonoid dihydrobiopterin (Fig. 1). Tetrahydrobiopterin also functions as a cofactor in the hydroxylation of tyrosine to dihydroxyphenylalanine (Lloyd and Weiner, 1971) and of tryptophan to 5-hydroxytryptophan (Hosoda and Glick, 1966). A defect in tetrahydrobiopterin metabolism would therefore impair the synthesis of the neurotransmitters, dopamine, noradrenaline, adrenaline, and serotonin in addition to interfering with the hydroxylation of phenylalanine.

Tetrahydrobiopterin can be quantitated by its effect on the growth of the haemoflagellate Crithidia fasciculata. An assay based on this principle has been used to measure biopterin derivatives in urine and it has been shown that the principal pteridine derivatives assayed are 7,8-dihydrobiopterin and 5, 6, 7, 8-tetrahydrobiopterin (Leeming and Blair, 1974). This assay has subsequently been modified so it can be applied to plasma (Leeming, et al., 1976b).

The present study is concerned with the assay of plasma biopterin derivatives in normal subjects and in a group of patients with phenylketonuria. The 


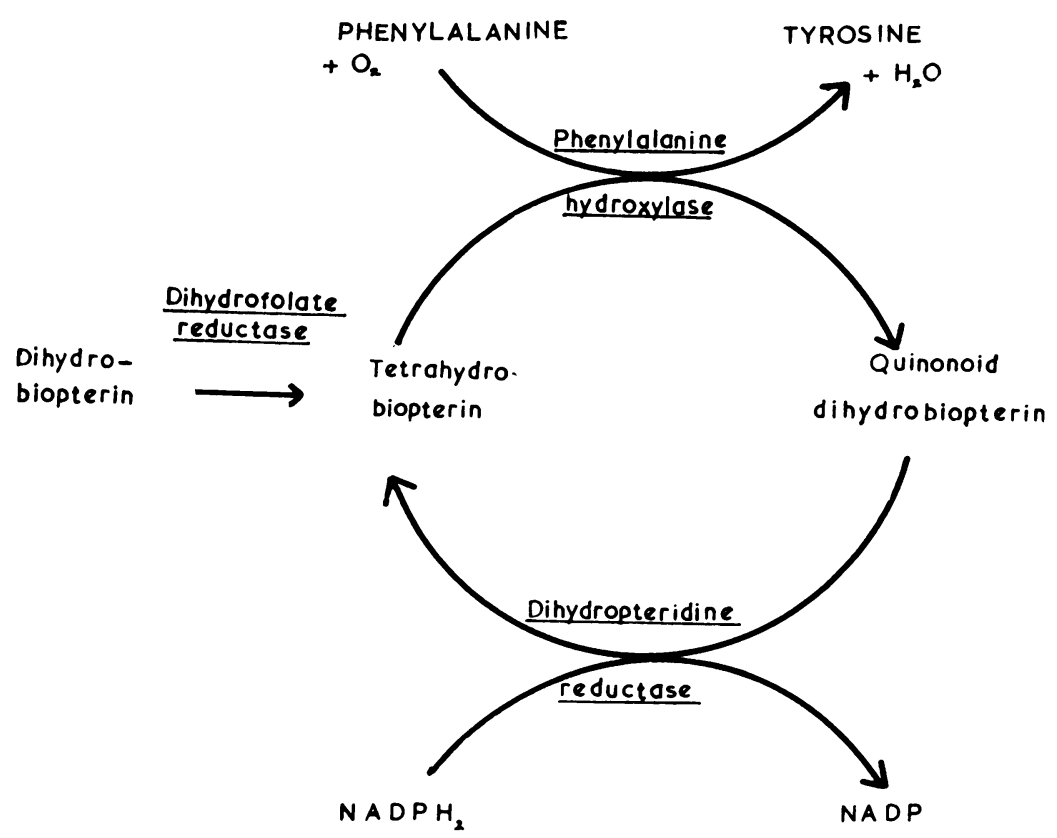

FIG. 1.-Hydroxylation of phenylalanine to tyrosine showing the inter-relation of the biopterin derivatives.

discovery of a significantly different 'crithidia factor' concentration in these two groups led to further experiments designed to elucidate its cause.

\section{Patients}

Phenylketonuric patients. 21 patients at Birmingham Children's Hospital aged 6 months to 16 years, 18 of whom were maintained on a low phenylalanine diet, were studied. In 2 children, aged 9 and 16 years, dietary control of phenylalanine intake had been discontinued 1 year and 2 years earlier respectively, and the remaining child proved to have transient hyperphenylalaninaemia associated with increased plasma tyrosine concentration. 36 specimens of capillary or venous blood with not more than 4 specimens from any individual were obtained from this group. In one newly diagnosed patient specimens were obtained before and during dietary control.

Control subjects. 10 children, aged 1 month to 3 years, attending hospital to have blood taken for other investigations, and who did not have phenylketonuria, were used as controls.

\section{Methods}

Plasma phenylalanine, tyrosine, tryptophan, and biopterin derivatives were measured. The determination of phenylalanine, tyrosine, and tryptophan was carried out by ion exchange chromatography using a Technicon amino acid analyser (TSM) after depro- teinizing heparinized plasma with sulphosalicylic acid (50 $\mathrm{mg} / \mathrm{ml}$ plasma) Technicon (1971). An accelerated programme was used for tyrosine and phenylalanine using the method of Cooke and Raine (1970). Plasma biopterin derivatives were determined using a Crithidia fasciculata assay by a modification (Leeming et al., 1976b) of the method of Leeming and Blair (1974).

\section{Results}

The Table shows the mean plasma concentration of biopterin derivatives in phenylketonuric patients who were on a phenylalanine-restricted diet and in normal children. The concentration of biopterin derivatives was significantly increased $(P<0.001)$ in the phenylketonuric group. Preliminary examination of the data suggested that in phenylketonuric patients there was a correlation between plasma biopterin derivates and phenylalanine concentrations. (These data together with all subsequent data obtained in further investigations are presented in Fig. 4 and will be discussed later.) This observation was supported by the fact that when biopterin derivatives were determined in a phenylketonuric patient before and after the phenylalanine-restricted diet was started the biopterin derivatives concentration decreased in parallel with the phenylalanine concentration (Fig. 2). In 3 other phenylketonuric patients for whom 
TABLE

Plasma biopterin derivatives, phenylalanine and tyrosine concentrations in normal and phenylketonuric children.

\begin{tabular}{|c|c|c|}
\hline & Phenylketonuric children & Normal children \\
\hline $\begin{array}{l}\text { No. of determinations } \\
\text { Biopterin derivatives }(\mu \mathrm{g} / \mathrm{l}) \\
\text { Range } \\
\text { Mean } \pm 1 \text { SE }\end{array}$ & $\begin{array}{c}30 \\
1 \cdot 3-13 \cdot 3 \\
4 \cdot 86 \pm 0 \cdot 51\end{array}$ & $\begin{array}{c}10 \\
1 \cdot 1-3 \cdot 7 \\
1 \cdot 78 \pm 0 \cdot 25\end{array}$ \\
\hline $\begin{array}{l}\text { Phenylalanine }(\mathrm{mmol} / \mathrm{l}) \\
\text { Range } \\
\text { Mean } \pm 1 \mathrm{SE}\end{array}$ & $\begin{array}{c}0.13-2 \cdot 36 \\
0.771 \pm 0.102\end{array}$ & $\begin{array}{c}0.3-0.09 \\
0.054 \pm 0.007\end{array}$ \\
\hline $\begin{array}{l}\text { Tyrosine }(\mathrm{mmol} / \mathrm{l}) \\
\text { Range } \\
\text { Mean } \pm 1 \mathrm{SE}\end{array}$ & $\begin{array}{c}0.03-0.09 \\
0.068 \pm 0.008\end{array}$ & $\begin{array}{c}0.06-0.22 \\
0 \cdot 114 \pm 0.016\end{array}$ \\
\hline
\end{tabular}

Conversion: SI to traditional units-Phenylalanine: $1 \mathrm{mmol} / \mathrm{l} \approx 16.5 \mathrm{mg} / 100 \mathrm{ml}$. Tyrosine: $1 \mathrm{mmol} / 1 \approx 18 \mathrm{mg} / 100 \mathrm{ml}$.

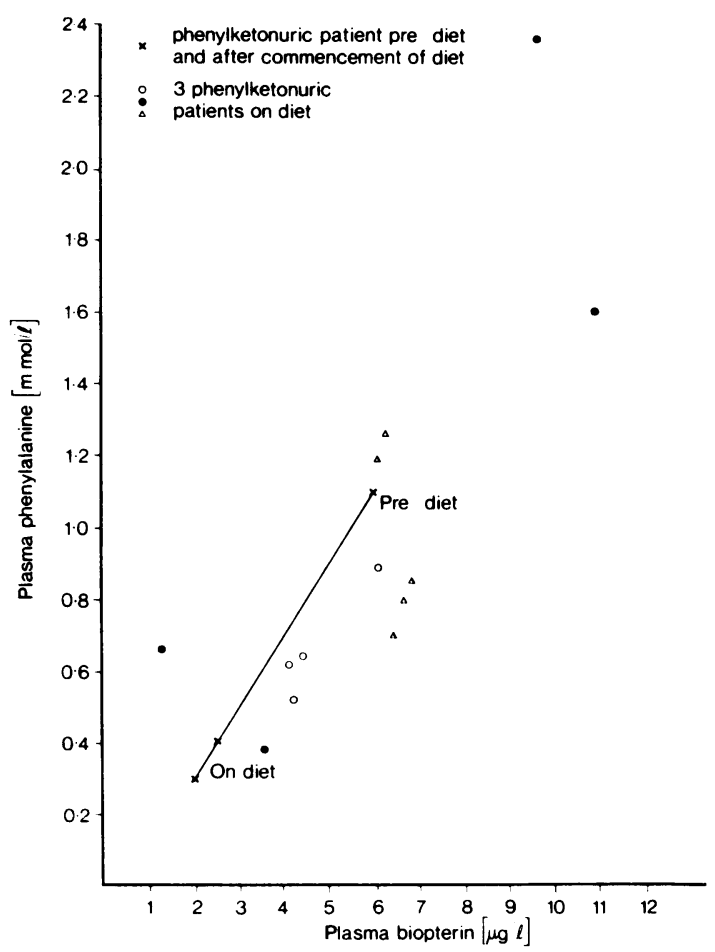

Fig. 2.-Relation of plasma phenylalanine and biopterin derivatives in 4 phenylketonuric patients. Conversion: $S I$ to traditional units-Phenylalanine: $1 \mathrm{mmol} / \mathrm{l} \approx$ $16.5 \mathrm{mg} / 100 \mathrm{ml}$.

plasma concentrations of phenylalanine and biopterin derivatives were available (also shown in Fig. 2) the same correlation was obtained, though the scatter is wider than in the patient studied immediately before and during treatment.

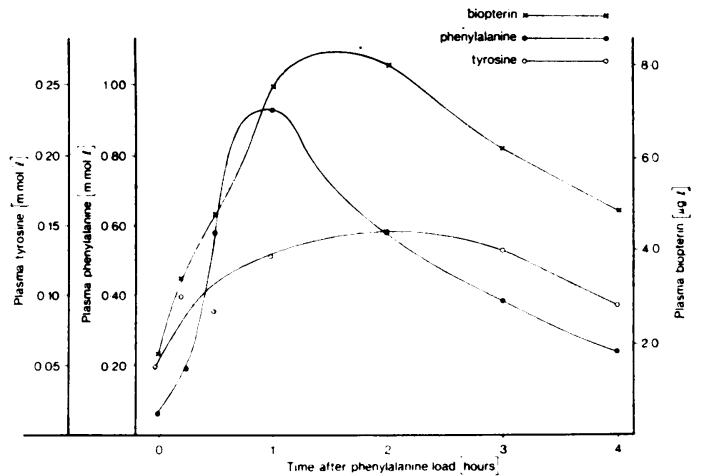

FIG. 3.-Mean plasma phenylalanine, tyrosine, and biopterin derivatives concentrations in 7 normal adults after a $7 \mathrm{~g} \mathrm{~L}$-phenylalanine load. Conversion: SI to traditional units-Tyrosine: $1 \mathrm{mmol} / \mathrm{l} \approx 18 \mathrm{mg} / 100 \mathrm{ml}$.

Correlation of plasma biopterin derivatives with phenylalanine suggests that phenylalanine, or a metabolite of it, may control biopterin concentration.

Oral phenylalanine, tyrosine, and tryptophan loads. The effect of raised plasma phenylalanine levels on plasma biopterin derivatives was therefore studied and, since tetrahydrobiopterin is required in the metabolism of tyrosine and tryptophan, the effect of these two amino acids was also studied. There is little occasion to load nonphenylketonuric children with phenylalanine and therefore these studies were made in normal adults. After an overnight fast 7 subjects were given $7 \mathrm{~g}$ oral L-phenylalanine and blood was taken before and hourly for 4 hours after the load. In 2 subjects specimens were taken at 15 and 30 minutes after the dose. Further studies were made in 3 subjects 
using 1, 2, and $4 \mathrm{~g}$ L-phenylalanine, and in one subject $7 \mathrm{~g}$ L-tyrosine, and in 2 subjects $7 \mathrm{~g} \mathrm{~L}$ tryptophan were given.

As a result of the phenylalanine loading studies a series of plasma biopterin derivatives and their related phenylalanine and tyrosine concentrations were obtained. The mean values obtained with the 7 subjects who received $7 \mathrm{~g} \mathrm{~L}$-phenylalanine are shown in Fig. 3, and the interpretation of these results will be discussed later. Phenylalanine and biopterin derivatives concentrations in these adults, together with the series obtained in the phenylketonuric patients who were not subjected to a phenylalanine load are shown in Fig. 4. It is

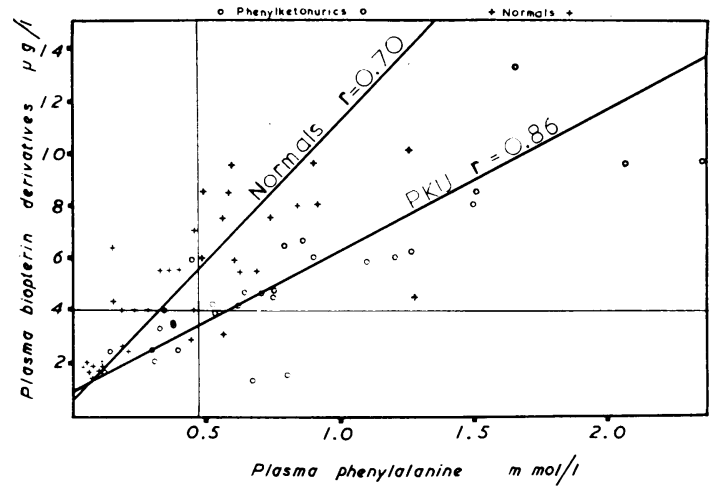

FIG. 4.-Relation of plasma phenylalanine and biopterin derivatives in phenylketonuric patients and in normal adults after phenylalanine loading.

clear that in both groups concentration of biopterin derivatives increases with that of phenylalanine but that the rate of increase is different $(P<0.05$, $\chi^{2}$ test).

In contrast to measuring the response of biopterin derivatives to increased phenylalanine concentration, a normal adult was given a phenylalanine-free diet for 11 days to see if reducing plasma phenylalanine might result in a fall in plasma biopterin derivatives. Plasma biopterin derivatives, phenylalanine, and tyrosine concentrations were determined. Plasma phenylalanine fell from 0.05 to $0.01 \mathrm{mmol} / 1 \quad(0 \cdot 8-0 \cdot 17 \mathrm{mg} / 100 \mathrm{ml})$ and was accompanied by a significant reduction of plasma biopterin derivatives $(\mathrm{P}<0.01-0.02)$ (Fig. 5). There was no corresponding reduction in tyrosine concentration.

The possibility that the effect of phenylalanine on plasma biopterin derivatives is an effect of phenylalanine or a metabolite on the biopterin derivatives assay was examined by adding serial dilutions of phenylalanine, tyrosine, phenylacetic acid, and

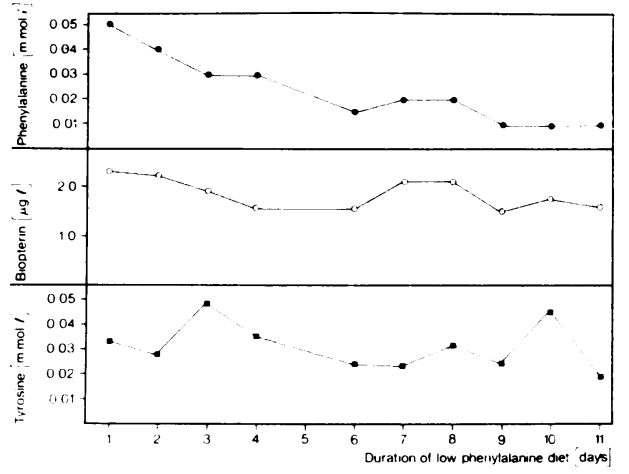

FIG. 5.-Effect of a low phenylalanine diet on plasma phenylalanine, biopterin derivatives, and tyrosine concentrations in a normal adult.

orthohydroxyphenyl acetic acid to the assay medium to give concentrations ranging from $125 \mathrm{pg} / \mathrm{ml}$ to $125 \mu \mathrm{g} / \mathrm{ml}$, both in the presence and absence of added biopterin. None of these compounds was active alone and none showed any synergistic activity with the added biopterin.

Because of the different relation between biopterin derivatives and phenylalanine in normal subjects and in phenylketonuric patients it was considered probable that a different biopterin derivative was present within these two groups. Plasma was chromatographed using three different solvent systems $(0.5 \%$ sodium carbonate, 3\% ammonium chloride, and 5\% acetic acid). In each case plasma was streaked across a thin layer cellulose plate $(20 \times 20 \mathrm{~cm})$ and this developed for $15 \mathrm{~cm}$ in one of the solvents in the dark. The cellulose was scraped from the plates in bands $1.5 \mathrm{~cm}$ broad, eluted with $0.5 \mathrm{~mol} / 1$ phosphate buffer $p \mathrm{H} 5 \cdot 0$, and the eluates assayed for biopterin derivatives. The chromatographic properties of the biopterin derivative in the plasma from 3 phenylketonuric patients and 3 normal subjects were identical in all three solvent systems and corresponded with those of 7,8 ,- dihydrobiopterin.

The oral tyrosine load did not produce any significant alteration in plasma biopterin derivatives or phenylalanine concentration over 4 hours (Fig. 6), nor did tryptophan cause any significant increase in concentration of biopterin derivatives over 4 hours in one subject or 24 hours in the other (Fig. 7).

Biopterin response in a patient with hyperphenylalaninaemia. During the first 3 weeks of life a neonate showed plasma phenylalanine concentrations of $0.75,0.87$, and $0.76 \mathrm{mmol} / 1$ $(12 \cdot 4,14 \cdot 4,12 \cdot 6 \mathrm{mg} / 100 \mathrm{ml})$ with slightly in- 


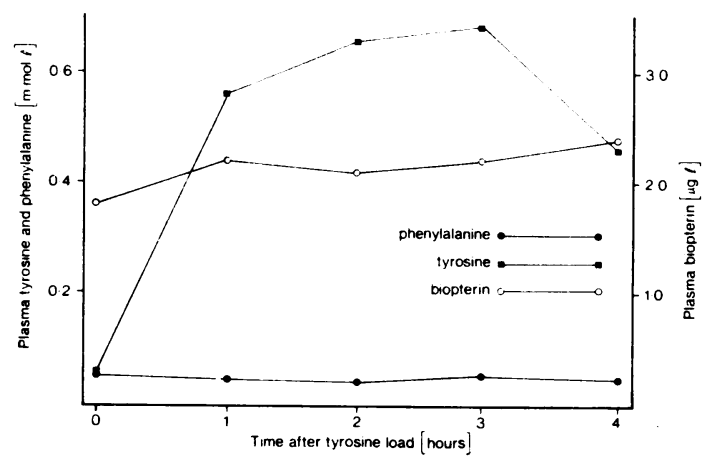

Fig. 6.-Plasma phenylalanine, tyrosine, and biopterin derivatives concentrations in a normal adult after a $7 \mathrm{~g}$ L-tyrosine load.



FIG. 7.-Plasma tryptophan in 1 and biopterin derivatives concentrations in 2 normal adults after $7 \mathrm{~g}$ L-tryptophan load.

creased corresponding tyrosine concentrations of $0 \cdot 17,0 \cdot 16$, and $0 \cdot 26 \mathrm{mmol} / 1(3 \cdot 1,2 \cdot 9,4 \cdot 7 \mathrm{mg} /$ $100 \mathrm{ml}$ ) respectively. Plasma concentration of biopterin derivatives in the third specimen was $4 \cdot 0 \mu \mathrm{g} / 1$. This degree of hyperphenylalaninaemia is not consistent with a diagnosis of classical phenylketonuria, and because the plasma phenylalanine concentrations were not excessive and could be expected to fall over the following week or two, the patient was not given a phenylalanine-restricted diet. At 2 months of age plasma phenylalanine had fallen to $0.12 \mathrm{mmol} / 1(2 \mathrm{mg} / 100 \mathrm{ml})$, and plasma biopterin derivatives had also fallen to within the normal range. At this stage the patient was subjected to an oral phenylalanine load $(0 \cdot 2 \mathrm{~g} / \mathrm{kg})$ to determine whether his condition should be regarded as a variant of phenylketonuria or as a case of hyperphenylalaninaemia. Phenylalanine increased to an abnormally high level at 2 hours, persisting over 4 hours (Fig. 8). Tyrosine did not
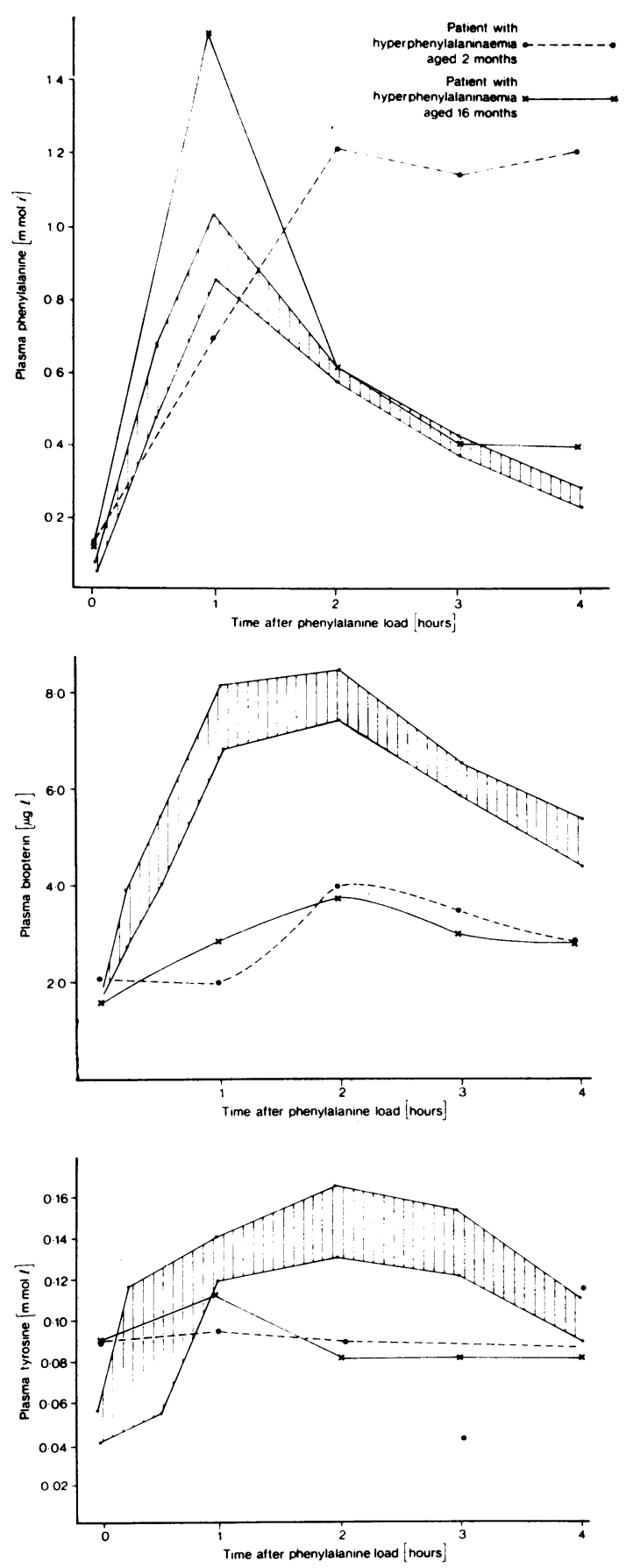

Fig. 8.-Plasma phenylalanine, tyrosine, and biopterin derivatives concentrations in a patient with hyperphenylalaninaemia after an oral load of $L$-phenylalanine $(0 \cdot 2$ $\mathrm{g} / \mathrm{kg}$ ). Hatched area represents normal mean $\pm 1 S E$. 
increase and biopterin derivatives showed only a slight rise at 2 hours. These studies were repeated when the child was aged 16 months having developed normally without treatment. Plasma tyrosine and biopterin derivatives response to the phenylalanine load remained abnormal in spite of some increased tolerance to the latter (Fig. 8).

\section{Discussion}

Pteridine coenzymes are known to be required for normal activity of a number of enzymes (Rembold and Gyure, 1972). The special importance of tetrahydrobiopterin in the action of phenylalanine hydroxylase, whose deficiency underlies phenylketonuria, has been highlighted recently in the discussion of clinical variants of this disorder (Smith et al., 1975; Bartholomé, 1974; Butler et al., 1975; Leeming et al., 1976a).

The present study shows that phenylketonuric patients, even on a phenylalanine-restricted diet, have raised plasma biopterin concentrations. The extent of this increase correlates with phenylalanine concentration, which is further illustrated by the progressive fall in biopterin concentration after phenylalanine restriction in a newly diagnosed phenylketonuric patient. A similar correlation between plasma phenylalanine and biopterin derivatives concentration occurs in normal adults given an oral load of phenylalanine. The more marked effect on biopterin derivatives of a given phenylalanine concentration in these circumstances may reflect the acute rise resulting from an amino acid load compared with the more constant phenylalanine stimulus present in the phenylketonuric patients. Alternatively, the different response may reflect the different kinetic properties of normal and mutant forms of phenylalanine hydroxylase resulting in a different relation between substrate and coenzyme (Kaufman and Fisher, 1974).

The peak value of plasma phenylalanine occurred 1 hour after the load followed by biopterin derivatives at 1.5 hours, and then tyrosine between 2 and 2.5 hours. This progression suggests that a rise in phenylalanine concentration is the stimulus for a rise in biopterin derivatives. Conversely, reduction in plasma phenylalanine, produced by phenylalanine restriction, was followed by a fall in plasma biopterin derivatives. The evidence suggests that plasma phenylalanine plays a role in the regulation of plasma concentration of biopterin derivatives. The concentration of biopterin derivates is significantly greater in tissue than in plasma (Baker et al., 1974; Leeming et al., 1976b). Phenylalanine may directly stimulate endogenous synthesis of biopterin derivatives, which has re- cently been shown to occur in mammalian liver (Fukushima et al., 1975), or may cause its release from tissue into the extracellular fluid and plasma. However, as phenylalanine concentration increases, the resulting increase in phenylalanine hydroxylase activity will result in an increased requirement for tetrahydrobiopterin and this too may trigger further synthesis of this coenzyme.

Tyrosine and tryptophan hydroxylases also involve pteridine coenzymes (Lloyd and Weiner, 1971; Hosoda and Glick, 1966), but in contrast to phenylalanine, giving these amino acids to normal adults promoted no increase of plasma biopterin derivatives comparable with that after phenylalanine. The major pathway for phenylalanine metabolism involves pteridine-dependent hydroxylation as the first step. However, while tyrosine and tryptophan hydroxylation is known to be the major pathway in certain organs, notably brain for tryptophan, and brain and adrenals for tyrosine, a major proportion of both amino acids follows pteridine-independent pathways (Guroff, 1972). Moreover, both tyrosine and tryptophan hydroxylases are inhibited by their substrates, much more than phenylalanine hydroxylase (Kaufman and Fisher, 1974), which may explain the observed differences in response.

The patient with hyperphenylalaninaemia responded abnormally to oral phenylalanine in that there was almost no increase in biopterin derivatives concentration. This suggests that the hyperphenylalaninaemia may be due to defective hydroxylation consequent upon a primary abnormality of some aspect of tetrahydrobiopterin metabolism or availability. Such defective utilization of cofactor has already been suggested to explain several cases of a new variant of phenylketonuria characterized by progressive neurological deterioration despite dietary restriction of phenylalanine (Bartholomé, 1974; Smith et al., 1975; Butler et al., 1975; Leeming et al., 1976a). In one case (Kaufman et al., 1975b) a deficiency of dihydropteridine reductase has been shown, and as this enzyme is required for both tyrosine and tryptophan hydroxylation, and hence for synthesis of dopamine, noradrenaline, adrenaline, and serotonin, disturbed neurotransmitter function may underlie the unusual neurological symptoms in these cases. However, this clinical variant has yet to be shown to be a single entity since in one patient dihydropteridine reductase, as well as the other known components of the phenylalanine hydroxylating system, were normal (Kaufman, Milstien, and Bartholomé, 1975a).

Treatment with biopterin derivatives is still the subject of controversy (Bartholomé and Byrd, 1975; Danks, Cotton, and Schlesinger, 1975; 
Kaufman, 1975; Smith et al., 1975), particularly as there is evidence that tetrahydrobiopterin does not readily enter the brain (Kettler, Bartholini, and Pletscher, 1974). It will be important to compare the concentrations of biopterin derivatives in these clinical variants with those in classical phenylketonuria.

The abnormal response of biopterin derivatives to phenylalanine in the hyperphenylalaninaemic case distinguishes this from patients with classical phenylketonuria, and may prove a worthwhile investigation to differentiate patients with these two different disorders at a much earlier age than is possible using present criteria.

We thank our clinical colleagues, and especially Dr. B. S. B. Wood, in whose charge are most of the phenylketonuric patients, for the opportunity to study these.

\section{REFERENCES}

Baker, M., Frank, O., Bacchi, C. J., and Hutner, S. H. (1974). Biopterin content of human and rat fluids and tissues determined protozoologically. American fournal of Clinical Nutrition, 27, 1247.

Bartholomé, K. (1974). A new molecular defect in phenylketonuria. Lancet, 2, 1580.

Bartholomé, K., and Byrd, D. J. (1975). L-dopa and 5-hydroxtryptophan therapy in phenylketonuria with normal phenylalanine hydroxylase activity. Lancet, 2, 1042.

Blakley, R. L. (1969). The Biochemistry of Folic Acid and Related Pteridines. North-Holland, Amsterdam.

Butler, I. J., Holtzman, N. A., Kaufman, S., Koslow, S. H., Krumholz, A., and Milstien, S. (1975). Phenylketonuria due to a deficiency of dihydropteridine reductase. Pediatric Research, $9,348$.

Cooke, J. R., and Raine, D. N. (1970). Accelerated determination of phenylalanine and tyrosine using the Technicon Sequential Multisample Analyser. Annals of Clinical Biochemistry, 7, 49.

Danks, D. M., Cotton, R. G. H., and Schlesinger, P. (1975). Tetrahydrobiopterin treatment of variant form of phenylketonuria. Lancet, 2, 1043.
Fukushima, K., Eto, I., Mayumi, T., Richter, W., Goodson, S., and Shiota, T. (1975). Biosynthesis of pterins in mammalian systems. Chemistry and Biology of Pteridines, p. 247 . Ed. by W. Pfleiderer de Gruyter, Berlin.

Guroff, G. (1971). Transport and metabolism of amino-acids. Basic Neurochemistry, p. 191. Ed. by R. W. Albers, G. J. Siegel, R. Katzman and B. W. Agranoff. Little, Brown, Boston.

Hosoda, S., and Glick, D. (1966). Properties of tryptophan hydroxylase from neoplastic murine mast cell. Fournal of Biological Chemistry, 241, 192.

Kaufman, S. (1975). Pterin administration as a therapy for PKU due to dihydropteridine-reductase deficiency? Lancet, 2 , 767.

Kaufman, S., and Fisher, D. B. (1974). Molecular Mechanisms of Oxygen Activation, p. 285. Ed. by O. Hayaishi. Academic Press, New York.

Kaufman, S., Milstien, S., and Bartholomé, K. (1975a). New forms of phenylketonuria. Lancet, 2, 708.

Kaufman, S., Holtzman, N. A., Milstien, S., Butler, I. J., and Krumholz, A. (1975b). Phenylketonuria due to a deficiency of dihydropteridine reductase. New England fournal of Medicine, 293, 785.

Kettler, R., Bartholini, G., and Pletscher, A. (1974). In vivo enhancement of tyrosine hydroxylation in rat striatum by tetrahydrobiopterin. Nature, 249, 476.

Leeming, R. J., and Blair, J. A. (1974). Crithidia factors in human urine. Biochemical Medicine, 11, 122.

Leeming, R. J., Blair, J. A., and Rey, F. (1976a). Biopterin derivatives in atypical phenylketonuria. Lancet, 1, 99.

Leeming, R. J., Blair, J. A., Melikian, V., and O'Gorman, D. (1976b). Biopterin derivatives in human body fluids and tissues. Fournal of Clinical Pathology, 29, 444.

Lloyd, T., and Weiner, N. (1971). Isolation and characterization of a tyrosine hydroxylase cofactor from bovine adrenal medulla. Molecular Pharmacology, 7, 569.

Rembold, H., and Gyure, W. L. (1972). Biochemistry of the pteridines. Angewandte Chemie, International Edition, 11, 1061.

Smith, I., Clayton, B. E., and Wolff, O. H. (1975). New variant of phenylketonuria with progressive neurological illness unresponsive to phenylalanine restriction. Lancet, 1, 1108.

Technicon Instruments Co. Ltd. (1971). Operation Manual for the Technicon TSM System. Technical Publication No. TAI-023300 . Tarry Town, New York.

Correspondence to Dr. D. N. Raine, Department of Clinical Chemistry, Children's Hospital, Ladywood Middleway, Birmingham B16 8ET. 